\title{
Continuum of Zero Points of a Mapping on a Compact, Convex Set ${ }^{1}$
}

\author{
A.J.J. Talman ${ }^{2}$ and Y. Yamamoto ${ }^{3}$
}

August 2001

\begin{abstract}
Let $X$ be a convex, compact, nonempty set in $\mathbb{R}^{n}$ and $\phi$ an upper semicontinuous mapping from $X$ to the collection of nonempty, convex, compact subsets of $\mathbb{R}^{n}$. We show that for any nonzero vector $c$ in $\mathbb{R}^{n}$ there exists a connected set of stationary points of $\phi$ on $X$ with respect to $c$ containing a point in the boundary of $X$ at which $c^{\top} x$ is minimized on $X$ and another point in the boundary of $X$ at which $c^{\top} x$ is maximized on $X$. We give several conditions on $\phi$, under which there exists a continuum of zero points of $\phi$ connecting two such points in the boundary of $X$. We also provide intersection results on a convex, compact set, where there exists a connected set of intersection points containing two distinct points in the boundary. Finally, we propose a simplicial variable dimension algorithm to approximate the connected set. The algorithm traces a piecewise linear path of points by making a sequence of linear programming pivoting steps in a system of $n+1$ equations. Each point on the path is a stationary point for the vector $c$ of a piecewise linear approximation of the mapping with respect to some simplicial subdivision of a polytope containing the set $X$.
\end{abstract}

Keywords: stationary point, continuum of zero points, simplicial algorithm, system of nonlinear equations, variational inequality, intersection points.

\footnotetext{
${ }^{1}$ The research of the first author has been made possible by a fellowship of the Japanese Society of the Promotion of Sciences. He thanks the society and also the University of Tsukuba for their hospitality. The second author is supported by the Grant-in-Aid for Scientific Research C(2) 13650061 of the Ministry of Education, Culture, Sports, Science and Technology of Japan.

${ }^{2}$ Dolf Talman, Department of Econometrics \& Operations Research and CentER, Tilburg University, P.O. Box 90153, 5000 LE Tilburg, The Netherlands. E-mail: talman@kub.nl

${ }^{3}$ Yoshitsugu Yamamoto, Institute of Policy and Planning Sciences, University of Tsukuba, Tsukuba, Ibaraki 305-8573, Japan. E-mail: yamamoto@shako.sk.tsukuba.ac.jp
} 


\section{Introduction}

Whenever a mathematical model of some phenomenon is constructed, for instance in engineering or in economics, the first question to ask is whether a solution to the model exists. A very powerful tool that is used to this end is Brouwer's fixed point theorem; see Brouwer (1912). When the model is not a system of equations but a system of correspondences, Kakutani's fixed point theorem (1941) is invoked. An alternative to fixed point theorems consists of using intersection theorems on polytopes, with the KKM theorem of Knaster, Kuratowski and Mazurkiewicz (1929) perhaps the most prominent example. It is well-known that there is a close relationship between fixed point theorems and intersection theorems. Yet another alternative consists of results that claim the existence of solutions to variational inequality problems, the existence of stationary points, or the existence of zero points.

For certain models, it is not only important to know that there exists at least one solution, but one would like to show the existence of a continuum of solutions. In economics the existence of a continuum of solutions leads to difficulties in expectation formation of agents, and as a consequence provides scope for endogenously generated fluctuations. A particular example comes from general equilibrium theory with price rigidities, where a continuum of zero points on the unit cube as a polytope is shown to exist in Herings (1998). It is therefore important to have generally applicable tools that guarantee the existence of a continuum of solutions to a certain system of equations.

This leads us to the following problem: Given a point-to-set mapping $\varphi: X \rightarrow \mathbb{R}^{n}$, with $X$ an arbitrary nonempty, convex, compact set, what reasonable conditions can guarantee the existence of a continuum of solutions to the system

$$
0^{n} \in \varphi(x) \text {. }
$$

Our approach to prove the existence of a continuum of solutions is to show that there is a connected subset of solutions that links together two distinct points in $X$, thereby guaranteeing the continuum.

It is well-known that under certain conditions a point-to-set mapping defined on a nonempty, convex, compact set has a solution to the variational inequality or stationary point problem, e.g. see Eaves (1971). In this paper we generalize this problem and define with respect to some given nonzero vector $c$ a parametric stationary point problem. We show that under the same conditions a point-to-set mapping defined on a nonempty, convex, compact set has a connected set of solutions to the parametric stationary point problem, called parameterized stationary points or stationary points with respect to $c$. The connected set contains two distinct points in the boundary of $X$. At one of these points, denoted by $x^{-}$, the value $c^{\top} x$ is minimized for $x \in X$, while at the other point, denoted by $x^{+}$, the value $c^{\top} x$ is maximized for $x \in X$. We give several conditions under 
which there exists a connected set of zero points linking two distinct boundary points of $X$.

Intersection results with a continuum of intersection points can be found in Freidenfelds (1974) on the unit simplex and Herings and Talman (1998) on the unit cube. We provide sufficient conditions for a collection of closed sets covering a nonempty, convex, compact set to have a connected set of intersection points containing two distinct points in the boundary of the set.

We also propose a simplicial variable dimension algorithm to approximate a connected set of zero points or parameterized stationary points on a compact, convex set. This type of algorithm was initiated by Scarf (1967). Simplicial homotopy methods were developed by Eaves (1972). The simplicial variable dimension restart algorithm was introduced by van der Laan and Talman (1979) to compute a fixed point of a continuous function from the unit simplex into itself. Such an algorithm generates a unique sequence of simplices of varying dimension in a simplicial subdivision of the underlying set and connects the arbitrarily chosen starting point with an approximate solution. For other recent developments, we refer to Talman and Yamamoto (1989), Yamamoto (1993), Brown, DeMarzo and Eaves (1996), DeMarzo and Eaves (1996), Yang (1996, 1999), and van der Laan, Talman and Yang (1998). Allgower and Georg (1990), Todd (1976), and Yang (1999) provide comprehensive treatments of simplicial algorithms.

In this paper we use the simplicial algorithm of Herings, Talman and Yang (2001) on polytopes to generate a finite sequence of simplices of varying dimension within a simplicial subdivision of a polytope containing the set $X$. This sequence connects two different simplices, one simplex in a face of the polytope containing $x^{-}$and the other one in a face of the polytope containing $x^{+}$. The sequence of simplices connecting these two simplices is generated by the algorithm by making a sequence of semi-lexicographic pivot steps in a linear system of $n+1$ equations. Induced by the sequence of adjacent simplices, the algorithm yields a piecewise linear path of parameterized stationary points of a piecewise linear approximation of the underlying mapping. When the mesh size of the simplicial subdivision of the polytope goes to zero and the sequence of polytopes converges to $X$, the sequence (or at least a subsequence) of piecewise linear paths converges to a connected set of parameterized stationary points of the original mapping.

The results in the paper generalize earlier results of Browder (1960), Mas-Colell (1974), and Herings, Talman and Yang (2001). In case of Browder's theorem the compact, convex set is the cartesian product of a compact, convex set of one dimension less and the unit interval $[0,1]$, while $c$ is the unit vector with the one on the last position. Mas-Colell's result is an extension of Browder's result to deal with point-to-set mappings. Both Browder and Mas-Colell proved their results via a rather sophisticated machinery. In Herings, Talman and Yang (2001) the compact, convex set is a polytope. In Browder's theorem and in 
Mas-Colell's theorem a connected set of fixed points is obtained connecting the levels 0 and 1 , whereas the result on the polytope yields a connected set of zero points connecting two different faces of the polytope.

This paper is organized as follows. In Section 2 we state the problem and give a general existence result. In Section 3 we give sufficient conditions for the existence of a connected set of zero points of a mapping. Section 4 states the intersection results and in Section 5 we discuss a simplicial algorithm.

\section{The Problem}

Consider an arbitrary nonempty, convex, compact set $X$ in $\mathbb{R}^{n}$ and let $c$ be an arbitrary nonzero vector in $\mathbb{R}^{n}$. Without loss of generality we assume that $X$ is full-dimensional and that $\|c\|_{2}=1$, where $\|\cdot\|_{2}$ denotes the Euclidean norm. Let $X^{-}$and $X^{+}$be defined by

$$
X^{-}=\left\{x^{-} \in X \mid c^{\top} x^{-}=\min _{x \in X} c^{\top} x\right\}
$$

and

$$
X^{+}=\left\{x^{+} \in X \mid c^{\top} x^{+}=\max _{x \in X} c^{\top} x\right\} .
$$

Since $X$ is compact, convex and full-dimensional, $X^{-}$and $X^{+}$are disjoint and both sets are nonempty, convex and compact. For $\alpha \in \mathbb{R}$, let $X_{\alpha}=\left\{x \in X \mid c^{\top} x=\alpha\right\}$, then for $\alpha^{-}=\min _{x \in X} c^{\top} x$ and $\alpha^{+}=\max _{x \in X} c^{\top} x$ we have that $\alpha^{+}>\alpha^{-}, X_{\alpha^{-}}=X^{-}$, and $X_{\alpha^{+}}=X^{+}$. Without loss of generality we assume that $\alpha^{-}=0$ and $\alpha^{+}=1$. For any $\alpha \in[0,1]$, the set $X_{\alpha}$ is a nonempty, convex, compact set. We first show that $X_{c^{\top} x}$ is continuous as a point-to-set mapping of the variable $x$ as long as $0 \leq c^{\top} x \leq 1$. Let $H(\alpha)$ be defined by $H(\alpha)=\left\{x \in \mathbb{R}^{n} \mid c^{\top} x=\alpha\right\}$ for any $\alpha \in[0,1]$, so $X_{c^{\top} x}=X \cap H\left(c^{\top} x\right)$. Let $H=\bigcup_{0 \leq \alpha \leq 1} H(\alpha)$. For $x \in H$, let $S(x)$ be defined by

$$
S(x)=X \cap H\left(c^{\top} x\right) \text {. }
$$

Lemma 2.1 $S$ is a continuous point-to-set mapping on $H$.

Proof: To prove that $S$ is a continuous mapping we prove that $S$ is both upper semicontinuous and lower semicontinuous on $H$. Let $\left\{x^{k}\right\}_{k \in \mathrm{N}}$ be a sequence of points in $H$ converging to $\bar{x} \in H$ and let $\left\{y^{k}\right\}_{k \in \mathrm{N}}$ be a sequence of points converging to some $\bar{y}$ such that $y^{k} \in S\left(x^{k}\right)$ for any $k \in \mathbb{N}$. Clearly, $\bar{y} \in X$ and $c^{\top} x^{k} \rightarrow c^{\top} \bar{x}$. Since $c^{\top} x^{k}=c^{\top} y^{k}$, for all $k \in \mathbb{N}$, we also have that $c^{\top} \bar{x}=c^{\top} \bar{y}$. Hence, $\bar{y} \in H\left(c^{\top} \bar{x}\right)$ and $S$ is upper semicontinuous on $H$.

To prove lower semicontinuity on $H$, take any sequence $\left\{x^{k}\right\}_{k \in \mathbf{N}}$ in $H$ converging to some $\bar{x} \in H$ and take any $\bar{y} \in S(\bar{x})$. We have to construct a sequence $\left\{y^{k}\right\}_{k \in \mathbf{N}}$ in $X$ such 
that $y^{k}$ converges to $\bar{y}$ and $y^{k} \in S\left(x^{k}\right)$ for every $k \in \mathbb{N}$. Take any $y^{-}$in $X^{-}$and $y^{+}$in $X^{+}$. For $k \in \mathbb{N}$, let

$$
\lambda^{k}=\left(c^{\top} \bar{y}-c^{\top} x^{k}\right) / c^{\top} \bar{y} \text { if } c^{\top} x^{k}<c^{\top} \bar{y} \text { or } c^{\top} \bar{y}=1
$$

and

$$
\lambda^{k}=\left(c^{\top} x^{k}-c^{\top} \bar{y}\right) /\left(1-c^{\top} \bar{y}\right) \text { if } c^{\top} x^{k} \geq c^{\top} \bar{y} \text { and } c^{\top} \bar{y}<1 .
$$

Next, for $k \in \mathbb{N}$, let

$$
y^{k}=\left(1-\lambda^{k}\right) \bar{y}+\lambda^{k} y^{-} \text {if } c^{\top} x^{k}<c^{\top} \bar{y} \text { or } c^{\top} \bar{y}=1
$$

and

$$
y^{k}=\left(1-\lambda^{k}\right) \bar{y}+\lambda^{k} y^{+} \text {if } c^{\top} x^{k} \geq c^{\top} \bar{y} \text { and } c^{\top} \bar{y}<1 .
$$

Clearly, $\lambda_{k} \geq 0$ for every $k \in \mathbb{N}$, and, since $c^{\top} x^{k} \rightarrow c^{\top} \bar{y}$, we have that $\lambda^{k} \rightarrow 0$. So, for sufficiently large $k, 0 \leq \lambda^{k} \leq 1$ and therefore $y^{k} \in X$. Moreover, $c^{\top} y^{k}=c^{\top} x^{k}$ for all $k \in \mathbb{N}$. Hence, $y^{k} \rightarrow \bar{y}$ and for sufficiently large $k$ it holds that $y^{k} \in X \cap H\left(c^{\top} x^{k}\right)=S\left(x^{k}\right)$.

For $x \in X_{\alpha}, 0 \leq \alpha \leq 1$, let $G(x)$ be the subgradient of $X_{\alpha}$ at $x$, i.e.,

$$
G(x)=\left\{y \in \mathbb{R}^{n} \mid x^{\prime \top} y \leq x^{\top} y \text { for any } x^{\prime} \in X_{\alpha}\right\} .
$$

Lemma 2.2 $G$ is an upper semicontinuous point-to-set mapping on $X$ and $G(x)$ is a nonempty convex cone for any $x \in X$.

Proof: Suppose $x^{k} \rightarrow x, y^{k} \in G\left(x^{k}\right)$ for all $k$ and $y^{k} \rightarrow y$, and let $x^{\prime} \in X_{c^{\top} x}$. Since $c^{\top} x^{k} \rightarrow c^{\top} x$ and according to Lemma $2.1 X_{c^{\top} x}$ is continuous in $x$, there is a sequence $x^{\prime k} \in X_{c^{\top} x^{k}}$ such that $x^{\prime k} \rightarrow x^{\prime}$. By the choice of $y^{k}$ we have $\left(x^{k}\right)^{\top} y^{k} \geq\left(x^{\prime k}\right)^{\top} y^{k}$ for all $k$. Therefore $x^{\top} y \geq\left(x^{\prime}\right)^{\top} y$, and so $y \in G(x)$. That $G(x)$ is a nonempty convex cone for every $x$ follows from the fact that $X$ is a convex set.

Moreover we have the next lemma.

Lemma 2.3 If $y \in G(x)$, then also $y+\beta c \in G(x)$ for any $\beta \in \mathbb{R}$.

Proof: Take any $y \in G(x)$ and let $\alpha=c^{\top} x$. By definition $x^{\prime \top} y \leq x^{\top} y$ for any $x^{\prime} \in X_{\alpha}$. Hence, for any $\beta \in \mathbb{R}$,

$$
x^{\prime \top}(y+\beta c)=x^{\prime \top} y+\beta x^{\prime \top} c=x^{\prime \top} y+\beta \alpha \leq x^{\top} y+\beta x^{\top} c=x^{\top}(y+\beta c),
$$

for any $x^{\prime} \in X_{\alpha}$. Therefore, $y+\beta c$ lies in $G(x)$.

Next, let $\phi$ be an upper semicontinuous, bounded point-to-set mapping from $X$ to the collection of nonempty, convex, compact subsets of $\mathbb{R}^{n}$. A stationary point of $\phi$ on $X$ with respect to $c$ is defined as follows. 
Definition 2.4 A point $x^{*} \in X$ is a stationary point of the correspondence $\phi$ on $X$ with respect to the vector $c$ if there exists $f \in \phi\left(x^{*}\right)$ such that $\left(x^{*}-x\right)^{\top} f \geq 0$ for all $x \in X_{\alpha}$, where $\alpha=c^{\top} x^{*}$, i.e., $\phi\left(x^{*}\right) \cap G\left(x^{*}\right) \neq \emptyset$. A point $x^{*} \in X$ is a stationary point of the correspondence $\phi$ on $X$ if there exists $f \in \phi\left(x^{*}\right)$ such that $\left(x^{*}-x\right)^{\top} f \geq 0$ for all $x \in X$. A point $x^{*} \in X$ is a zero point of $\phi$ if $0^{n} \in \phi\left(x^{*}\right)$.

Clearly, every zero point of $\phi$ is a stationary point of $\phi$ on $X$ and every stationary point of $\phi$ on $X$ is a stationary point of $\phi$ on $X$ with respect to any nonzero vector $c$. We call the problem of finding a stationary point with respect to a nonzero vector $c$ a parametric variational inequality problem. A solution to it is also called a parameterized stationary point. Clearly, $x^{*}$ with $\alpha=c^{\top} x^{*}$ is a stationary point of $\phi$ on $X$ with respect to $c$ if and only if $x^{*}$ is a stationary point of $\phi$ on the set $X_{\alpha}$. Since the set $X_{\alpha}$ is nonempty, convex and compact, we know from Eaves (1971) that for any $\alpha \in[0,1]$ there exists a stationary point of $\phi$ on $X_{\alpha}$ and therefore there exists a stationary point $x$ of $\phi$ on $X$ with respect to $c$ satisfying $c^{\top} x=\alpha$. When varying $\alpha$ from 0 to 1 , we want to show that there exists a connected set of such points, having a nonempty intersection with both $X^{-}$and $X^{+}$and give conditions on $\phi$ under which there exists a connected set of zero points containing points in both these sets.

To show that there always exists a connected set of stationary points with respect to the vector $c$, we denote by $p(x), x \in H$, the orthogonal projection of $x$ on $X_{\alpha}$, where $\alpha=c^{\top} x$. Clearly, $x-p(x) \in G(p(x))$ for any $x \in H$. The next lemma shows that $p$ is a continuous function.

Lemma 2.5 The function $p$ from $H$ to $X$ is a continuous function.

Proof: By Lemma 2.1 we have seen that $S(x)=X \cap H\left(c^{\top} x\right)$ is continuous. Applying Corollary 8.1 of Hogan (1973) or Theorem 6 of Aubin and Cellina (1984) it follows that $p$ is a continuous function on $H$.

Using the results above we are able to prove the main result.

Theorem 2.6 Let $X$ be a full-dimensional, compact, convex set in $\mathbb{R}^{n}$, let $\phi$ be an upper semicontinuous, bounded, nonempty-, convex-, compact-valued point-to-set mapping from $X$ to $\mathbb{R}^{n}$, and let $c$ be an arbitrary nonzero vector in $\mathbb{R}^{n}$. Then there exists a connected set $C$ of stationary points of $\phi$ on $X$ with respect to $c$ such that $C \cap X^{-} \neq \emptyset$ and $C \cap X^{+} \neq \emptyset$.

Proof: Let $r$ be the orthogonal projection from $\mathbb{R}^{n}$ to $H(0)$, so $r$ is a linear function and for any $y \in \mathbb{R}^{n}$ it holds that $r(y)=y-\left(c^{\top} y\right) c$. Since $X$ is bounded and $\phi$ is bounded, the set

$$
Y=\{y \in H(0) \mid y=r(x+f), f \in \phi(x), x \in X\}
$$


is a bounded set in $\mathbb{R}^{n}$. Let $S$ be a compact, convex subset of $H(0)$ containing $Y$ in its relative interior and let the mapping $\psi: S \times[0,1] \rightarrow \mathbb{R}^{n}$ be defined by

$$
\psi(y, \alpha)=\left\{z \in \mathbb{R}^{n} \mid z=r(p(y+\alpha c)+f), f \in \phi(p(y+\alpha c))\right\} .
$$

Since $p$ is a continuous function, $r$ is a linear function and given the assumptions on $\phi$, for any $(y, \alpha) \in S \times[0,1]$ the set $\psi(y, \alpha)$ is a nonempty, convex and compact subset of $S$ and $\psi$ is upper semicontinuous and bounded on $S \times[0,1]$. With $S$ itself being a nonempty, convex, compact set, it follows from Mas-Colell (1975) that there exists a connected set $\bar{C}$ in $S \times[0,1]$ of fixed points of $\psi$ satisfying $\bar{C} \cap(S \times\{0\}) \neq \emptyset$ and $\bar{C} \cap(S \times\{1\}) \neq \emptyset$, where $(y, \alpha) \in S \times[0,1]$ is called a fixed point of $\psi$ if $y \in \psi(y, \alpha)$. Let $C^{\prime}=\left\{z \in \mathbb{R}^{n} \mid z=y+\alpha c,(y, \alpha) \in \bar{C}\right\}$, then $C^{\prime}$ is a connected set in $H$ satisfying $C \cap H(0) \neq \emptyset$ and $C \cap H(1) \neq \emptyset$ and $z \in C^{\prime}$ implies $y=r(p(z)+f)$ for some $f \in \phi(p(z))$ with $y \in S$ given by $y=z-\left(c^{\top} z\right) c$. Therefore, there exists $\alpha$ and $\beta$ in $\mathbb{R}$ satisfying

$$
z-\alpha c=p(z)+f-\beta c .
$$

Consequently,

$$
f=z-p(z)+(\beta-\alpha) c .
$$

Since $z-p(z) \in G(p(z))$, it follows from Lemma 2.3 that $f \in G(p(z))$ and so

$$
f \in \phi(p(z)) \cap G(p(z)) .
$$

This implies that $x=p(z)$ is a stationary point of $\phi$ on $X$ with respect to $c$. Finally, let the set $C$ be defined by

$$
C=\left\{x \in X \mid x=p(z), z \in C^{\prime}\right\}
$$

Since $p$ is a continuous function on $H$, we have that $C$ is a connected set. Moreover, $C^{\prime} \cap H(0) \neq \emptyset$ implies $C \cap X^{-} \neq \emptyset$ and $C^{\prime} \cap H(1) \neq \emptyset$ implies $C \cap X^{+} \neq \emptyset$. Also, $x \in C$ implies $x=p(z)$ for some $z \in C^{\prime}$, i.e., $x$ is a stationary point of $\phi$ on $X$ with respect to $c$.

The theorem says that for any given nonzero vector $c$ any Kakutani-type of mapping on a full-dimensional compact, convex set has a connected set of stationary points with respect to $c$ having a nonempty intersection with both the (extreme) set of $X$ at which $c^{\top} x$ is minimized on $X$ and the (extreme) set of $X$ on which $c^{\top} x$ is maximized on $X$. In the next section we discuss when a continuum of zero points exists. 


\section{Continuum of Zero Points}

In this section we give sufficient conditions under which there exists a connected set of zero points of a mapping on a nonempty, convex, compact set, connecting two distinct points in the boundary of the set. In the previous section we proved that there always exists a connected set of stationary points with respect to some nonzero vector. Clearly, if all these points are zero points of the mapping we obtain a continuum of zero points.

Theorem 3.1 Let $X, \phi, c$ satisfy the conditions of Theorem 2.6. If for any $x \in X$ it holds that

$$
\phi(x) \cap G(x) \subset\left\{0^{n}\right\},
$$

then there exists a connected set $C$ of zero points of $\phi$ in $X$ such that $C \cap X^{-} \neq \emptyset$ and $C \cap X^{+} \neq \emptyset$.

Proof: From Theorem 2.6 it follows that there exists a connected set $C$ of stationary points of $\phi$ on $X$ with respect to $c$ satisfying $C \cap X^{-} \neq \emptyset$ and $C \cap X^{+} \neq \emptyset$. Take any $x \in C$, then $G(x) \cap \phi(x) \neq \emptyset$. Since $G(x) \cap \phi(x) \subset\left\{0^{n}\right\}$ this implies $G(x) \cap \phi(x)=\left\{0^{n}\right\}$ and so $0^{n} \in \phi(x)$. Consequently, $C$ is a connected set of zero points of $\phi$ in $X$ having a nonempty intersection with both $X^{-}$and $X^{+}$.

The condition in the theorem says that at any $x \in X$ no nonzero element of the image $\phi(x)$ is allowed to lie in the subgradient $G(x)$ of $X_{\alpha}$. Although the condition itself is rather weak it has to hold for every element in the image set. A sufficient condition that is much stronger but only has to hold for (at least) one element of the image set is the following one, where for $x \in X$ the set $G^{*}(x)=\left\{z \in \mathbb{R}^{n} \mid z^{\top} y \leq 0\right.$ for any $\left.y \in G(x)\right\}$ denotes the polar cone of $G(x)$.

Theorem 3.2 Let $X, \phi, c$ satisfy the conditions of Theorem 2.6. If for any $x \in X$ it holds that

$$
\phi(x) \cap G^{*}(x) \neq \emptyset,
$$

then there exists a connected set $C$ of zero points of $\phi$ in $X$ such that $C \cap X^{-} \neq \emptyset$ and $C \cap X^{+} \neq \emptyset$.

The proof of this theorem does not follow immediately from Theorem 2.6, because the mapping $G^{*}$ is in general not an upper semicontinuous mapping on $X$. To prove the theorem, let the set $Q$ be defined by

$$
Q=\left\{q \in \mathbb{R}^{n} \mid\|q-x\|_{2} \leq 1, c^{\top} q=c^{\top} x, x \in X\right\}
$$

and for $\alpha, 0 \leq \alpha \leq 1$, let

$$
Q_{\alpha}=\left\{q \in Q \mid c^{\top} q=\alpha\right\} .
$$


The relative interior and relative boundary of $Q_{\alpha}$ are denoted by $\operatorname{int} Q_{\alpha}$ and bd$Q_{\alpha}$, respectively.

Lemma 3.3 The set $Q$ is a convex, compact and full-dimensional subset of $H$ and for any $\alpha \in[0,1]$ the set $Q_{\alpha}$ contains $X_{\alpha}$ in its relative interior.

Proof: To show convexity of $Q$, take any two points $q^{1}$ and $q^{2}$ in $Q$. Let $x^{1}=p\left(q^{1}\right)$ and $x^{2}=p\left(q^{2}\right)$. Take $q(\lambda)=\lambda q^{1}+(1-\lambda) q^{2}$ for any $\lambda, 0 \leq \lambda \leq 1$. To show that $q(\lambda) \in Q$, let $x(\lambda)=\lambda x^{1}+(1-\lambda) x^{2}$. Since $X$ is convex, $x(\lambda)$ lies in $X$. Moreover,

$$
c^{\top} q(\lambda)=\lambda c^{\top} q^{1}+(1-\lambda) c^{\top} q^{2}=\lambda c^{\top} x^{1}+(1-\lambda) c^{\top} x^{2}=c^{\top} x(\lambda)
$$

and

$$
\begin{aligned}
& \|q(\lambda)-x(\lambda)\|_{2}=\left\|\lambda\left(q^{1}-x^{1}\right)+(1-\lambda)\left(q^{2}-x^{2}\right)\right\|_{2} \\
& \leq \lambda\left\|q^{1}-p\left(q^{1}\right)\right\|_{2}+(1-\lambda)\left\|q^{2}-p\left(q^{2}\right)\right\|_{2} \leq 1 .
\end{aligned}
$$

Therefore, $q(\lambda) \in Q$. The other properties follow immediately from the definition of $Q$.

For $q \in Q$, let $v(q)=q-p(q)$. Since $Q$ is a subset of $H$ and, according to Lemma 2.5, the function $p$ is continuous on $H$, the function $v$ is continuous on $Q$. Also, $\|v(q)\|_{2}=1$ if and only if $q \in \operatorname{bd} Q_{\alpha}$ with $\alpha=c^{\top} q,\|v(q)\|_{2}=0$ if and only if $q \in X$, and $c^{\top} v(q)=0$ for any $q \in Q$. Furthermore, for $q \in Q$, let

$$
B(q)=\left\{y \in \mathbb{R}^{n} \mid y=\mu v(q)+\beta c, \mu \geq 0, \beta \in \mathbb{R}\right\}
$$

with polar cone

$$
B^{*}(q)=\left\{z \in \mathbb{R}^{n} \mid z^{\top} y \leq 0 \text { for all } y \in B(q)\right\}
$$

and let $D(q)$ be the subgradient of $Q_{\alpha}$ at $q$, where $\alpha=c^{\top} q$.

Lemma 3.4 The following properties hold:

(i) $D(q) \subset B(q)$ for any $q$ in $Q$.

(ii) $B(q) \subset G(p((q))$ for any $q$ in $Q$.

(iii) The mapping $B^{*}$ is upper semicontinuous on $Q$.

Proof: For $q \in \operatorname{int} Q_{\alpha}, \alpha \in[0,1]$, the subgradient $D(q)$ is equal to the set $B=\left\{y \in \mathbb{R}^{n} \mid\right.$ $y=\beta c, \beta \in \mathbb{R}\}$. Clearly, this set is contained in $B(q)$. For $q \in \operatorname{bd} Q_{\alpha}, \alpha \in[0,1]$, let the set $R(q)$ be defined by

$$
R(q)=\left\{r \in R^{n} \mid c^{\top} r=\alpha,\|r-p(q)\|_{2} \leq 1\right\} .
$$


Then $R(q)$ is contained in $Q_{\alpha}$ and contains $q$. Hence, $v(q)$ is the only vector in $D(q)$ with length one and satisfying $c^{\top} v(q)=0$. Now take any $w \in D(q)$. According to Lemma 2.5 also $v=w-\left(c^{\top} w\right) c$ lies in $D(q)$. Since $c^{\top} v=0$, we must have $v=\mu v(q)$ for some $\mu \geq 0$. Therefore, $w=\mu v(q)+\beta c$ for some $\beta \in \mathbb{R}$, and so $w \in B(q)$. This proves property (i). Property (ii) follows from Lemma 2.5 and the fact that $v(q) \in G(p(q))$ for any $q \in Q$, see also Proposition 1 of Aubin and Cellina (1984). To prove property (iii), notice that $B(q)=B$ if $q \in X$ and

$$
B(q)=\left\{y \in \mathbb{R}^{n} \mid y=\mu v(q)+\beta c, \mu \geq 0, \beta \in \mathbb{R}\right\}
$$

if $q \in Q \backslash X$. Since $v$ is a continuous function on $Q$, it follows that the mapping $B^{*}$ is continuous at any point $q \notin \operatorname{bd} X$ and upper semicontinuous at any $q \in \operatorname{bd} X$.

Next, consider the closure of the graph $\left\{(q, y) \mid y \in \phi(p(q)) \cap G^{*}(p(q)), q \in Q\right\}$ in $\mathbb{R}^{n} \times$ $\mathbb{R}^{n}$ and let $\psi(q)$ be the convex hull of the image of $q$ in this graph. Then $\psi$ is an upper semicontinuous, bounded point-to-set mapping from the full-dimensional, compact, convex set $Q$ in $\mathbb{R}^{n}$ to the collection of nonempty, compact, convex subsets of $\mathbb{R}^{n}$. From Theorem 2.6 it follows that there exists a connected set of stationary points of $\psi$ on $Q$ with respect to the vector $c$. For $m \in \mathbb{N}$, let $I_{m}$ denote the set of integers $\{1, \cdots, m\}$.

Lemma 3.5 Let $q^{*}$ be a stationary point of $\psi$ on $Q$ with respect to $c$, then $x^{*}=p\left(q^{*}\right)$ is a zero point of $\phi$ in $X$.

Proof: Since $q^{*}$ is a stationary point of $\psi$ on $Q$ with respect to $c$, there exists $f \in \psi\left(q^{*}\right)$ satisfying $f \in D\left(q^{*}\right)$. The first property implies there exist $f^{i}$ and nonnegative $\lambda_{i}, i \in$ $I_{n+1}$, with $\sum_{i=1}^{n+1} \lambda_{i}=1$, such that $f=\sum_{i=1}^{n+1} \lambda_{i} f^{i}$ and for every $i \in I_{n+1}$ it holds that $f^{i}=\lim _{k} f^{i, k}$ for certain $f^{i, k} \in \phi\left(p\left(q^{i, k}\right)\right) \cap G^{*}\left(p\left(q^{i, k}\right)\right)$, for all $k \in \mathbb{N}$, where $\left\{q^{i, k}\right\}_{k \in \mathbb{N}}$ is a sequence of points in $Q$ converging to $q^{*}$. Since $p$ is a continuous function and $\phi$ is upper semicontinuous, it follows that $f^{i} \in \phi\left(x^{*}\right)$ for all $i \in I_{n+1}$, and, since $\phi$ is convex-valued, also that $f \in \phi\left(x^{*}\right)$. On the other hand, from property (ii) of Lemma 3.4 it follows for all $i$ and $k$ that

$$
B\left(q^{i, k}\right) \subset G\left(p\left(q^{i, k}\right)\right)
$$

and therefore

$$
G^{*}\left(p\left(q^{i, k}\right)\right) \subset B^{*}\left(q^{i, k}\right) .
$$

Hence, $f^{i, k} \in B^{*}\left(q^{i, k}\right)$ for all $i$ and $k$. Since $B^{*}$ is upper semicontinuous according to property (iii) of Lemma 3.4, we obtain that $f^{i} \in B^{*}\left(q^{*}\right)$ for all $i \in I_{n+1}$, and so, since $B^{*}\left(q^{*}\right)$ is convex, also that $f \in B^{*}\left(q^{*}\right)$. Finally, property (i) of Lemma 3.4 together with $f \in D\left(q^{*}\right)$ implies that $f \in B\left(q^{*}\right)$. Therefore, $f \in B\left(q^{*}\right) \cap B^{*}\left(q^{*}\right)$. Since the latter set only contains the $n$-vector of zeros, it follows that $f=0^{n}$ and so $0^{n} \in \phi\left(x^{*}\right)$. 
PROOF OF THEOREM 3.2: From Lemma 3.5 it follows that there exists a connected set $C^{\prime}$ of points in $Q$ satisfying $C^{\prime} \cap Q_{0} \neq \emptyset$ and $C^{\prime} \cap Q_{1} \neq \emptyset$ and $q \in C^{\prime}$ implies $0^{n} \in \phi(p(q))$. Now, let $C=\left\{x \in X \mid x=p(q), q \in C^{\prime}\right\}$. Since $p$ is a continuous function on $X$, the set $C$ is a connected set in $X$. Moreover, $C^{\prime} \cap Q_{0} \neq \emptyset$ implies $C \cap X^{-} \neq \emptyset$ and $C^{\prime} \cap Q_{1} \neq \emptyset$ implies $C \cap X^{+} \neq \emptyset$, whereas $x \in C$ implies $0^{n} \in \phi(x)$. Hence, $C$ is a connected set of zero points of $\phi$ in $X$ having a nonempty intersection with both $X^{-}$and $X^{+}$.

The next theorem is a combination of the two latter theorems. It relaxes the rather strong condition of Theorem 3.2 to hold for at least one element of every image set and adds a condition for all elements in every image set, which is a weaker condition than the one in Theorem 3.1. For $x \in X$ with $\alpha=c^{\top} x$, let

$$
G_{0}(x)=\left\{y \in \mathbb{R}^{n} \mid c^{\top} y=0, x^{\prime \top} y \leq x^{\top} y \text { for all } x^{\prime} \in X_{\alpha}\right\}
$$

and

$$
G_{0}^{*}(x)=\left\{z \in \mathbb{R}^{n} \mid z^{\top} y \leq 0 \text { for all } y \in G_{0}(x)\right\} .
$$

Theorem 3.6 Let $X, \phi, c$ satisfy the conditions of Theorem 2.6. If for any $x \in X$ it holds that

(i) $\phi(x) \cap\left(G_{0}^{*}(x) \cap G(x)\right) \subset\left\{0^{n}\right\}$,

(ii) $\phi(x) \cap G_{0}^{*}(x) \neq \emptyset$,

then there exists a connected set $C$ of zero points of $\phi$ such that $C \cap X^{-} \neq \emptyset$ and $C \cap X^{+} \neq \emptyset$.

Proof: Define the mapping $\phi^{\prime}$ on $X$ by

$$
\phi^{\prime}(x)=\left\{y \in \mathbb{R}^{n} \mid c^{\top} y=0, y+\beta c \in \phi(x) \text { for some } \beta \in \mathbb{R}\right\} .
$$

Clearly, $\phi^{\prime}$ is upper semicontinuous and bounded on $X$ and for every $x \in X$ the set $\phi^{\prime}(x)$ is nonempty, convex and compact. We will show that $\phi^{\prime}$ satisfies the condition of Theorem 3.2. Condition (ii) implies that there exists $z \in \phi(x) \cap G_{0}^{*}(x)$. Let $y$ be such that $y=z-\beta c \in \phi^{\prime}(x)$ for some $\beta \in \mathbb{R}$, so $c^{\top} y=0$. To prove that also $y \in G^{*}(x)$, take any $v \in G(x)$, then $w=v-\left(c^{\top} v\right) c$ lies in $G_{0}(x)$. Since $z \in G_{0}^{*}(x)$, we have that $z^{\top} w \leq 0$. But $z^{\top} w \leq 0$ implies $y^{\top} w \leq 0$ because $c^{\top} w=0$, and $y^{\top} w \leq 0$ implies $y^{\top} v \leq 0$ because $c^{\top} y=0$. Hence, $y \in G^{*}(x)$. Consequently, for any $x \in X$ it holds that $\phi^{\prime}(x) \cap G^{*}(x) \neq \emptyset$. From Theorem 3.2 it follows that there exists a connected set $C$ of zero points of $\phi^{\prime}$ in $X$ having a nonempty intersection with both $X^{-}$and $X^{+}$. We now show that every $x \in C$ is also a zero point of $\phi$. Take any $x \in C$, then $0^{n} \in \phi^{\prime}(x)$ and so there exists $\beta \in \mathbb{R}$ satisfying $\beta c \in \phi(x)$. We also have that $\mu c \in G_{0}^{*}(x) \cap G(x)$ for any $\mu \in \mathbb{R}$. Hence,

$$
\beta c \in \phi(x) \cap\left(G_{0}^{*}(x) \cap G(x)\right) .
$$

Condition (i) implies that $\beta=0$ and so $0^{n} \in \phi(x)$. Therefore, $C$ is a connected set of zero points of $\phi$ having a nonempty intersection with both $X^{-}$and $X^{+}$. 


\section{Intersection Theorems}

The results in Section 3 can be used to state intersection theorems on a convex, compact set, where the set of intersection points contains a connected set containing at least two distinct points in the boundary. Let $X$ be again a full-dimensional, convex, compact set in $\mathbb{R}^{n}$ and $c$ an arbitrary nonzero vector in $\mathbb{R}^{n}$. Suppose that $\left\{D^{j} \mid j \in I_{k}\right\}$, for some $k \in \mathbb{N}$, is a closed covering of $X$, i.e., every $D^{j}, j \in I_{k}$, is a closed (possibly empty) subset of $X$ and their union is equal to $X$. Let the set $B$ be again defined by $B=\left\{y \in \mathbb{R}^{n} \mid y=\beta c, \beta \in \mathbb{R}\right\}$. To every $D^{j}, j \in I_{k}$, a vector $d^{j} \in \mathbb{R}^{n}$ corresponds. For $I \subset I_{k}$, let $D(I)$ be the convex hull of $\left\{d^{j} \mid j \in I\right\}$, i.e.,

$$
D(I)=\left\{y \in \mathbb{R}^{n} \mid y=\sum_{j \in I} \lambda_{j} d^{j}, \lambda_{j} \geq 0, \sum_{j \in I} \lambda_{j}=1\right\} .
$$

The index set $I \subset I_{k}$ is said to be balanced if $D(I) \cap B \neq \emptyset$. A point $x \in X$ is said to be an intersection point if the set $I^{x}$ is balanced, where

$$
I^{x}=\left\{j \in I_{k} \mid x \in D^{j}\right\}
$$

i.e., a point $x$ in $X$ is an intersection point if the convex hull of the vectors $d^{j}$ for which $x$ lies in $D^{j}$ intersects the line $B$ and therefore contains a multiple of the vector $c$ or $-c$. For $I \subset I_{k}$, let $D_{0}(I)$ be defined by

$$
D_{0}(I)=\left\{y \in \mathbb{R}^{n} \mid y=r(z), z \in D(I)\right\},
$$

where $r$ is again the orthogonal projection on $H(0)$. Then $x$ is an intersection point if and only if $0^{n} \in D_{0}\left(I^{x}\right)$. Now we can use the zero points theorems of Section 3 to give sufficient conditions for the existence of a continuum of intersection points.

Theorem 4.1 Let $\left\{D^{j} \mid j \in I_{k}\right\}$ be a closed covering of a full-dimensional, convex, compact set $X$ in $\mathbb{R}^{n}$ and let $\left\{d^{j} \mid j \in I_{k}\right\}$ be a corresponding set of vectors in $\mathbb{R}^{n}$. Then there exists a connected set $C$ of intersection points in $X$ satisfying $C \cap X^{-} \neq \emptyset$ and $C \cap X^{+} \neq \emptyset$ if one of the following three conditions is satisfied:

1. $D\left(I^{x}\right) \cap G(x) \subset B$, for every $x \in X$.

2. $D_{0}\left(I^{x}\right) \cap G^{*}(x) \neq \emptyset$, for every $x \in X$.

3. $D\left(I^{x}\right) \cap\left(G_{0}^{*}(x) \cap G(x)\right) \subset B$ and $D_{0}\left(I^{x}\right) \cap G_{0}^{*}(x) \neq \emptyset$, for every $x \in X$.

Proof: The proof follows from the fact that if we define $\phi(x)=D_{0}\left(I^{x}\right), x \in X$, the mapping $\phi$ satisfies one of the three zero points theorems of Section 3 and therefore there 
exists a connected set $C$ of zero points of $\phi$ in $X$ having a nonempty intersection with both $X^{-}$and $X^{+}$. Clearly, a zero point of $\phi$ is an intersection point in $X$.

\section{The Algorithm}

To follow approximately a connected set of stationary points of a mapping on a convex, compact set $X$ with respect to some nonzero vector $c$, we approximate the latter set by a sequence of polytopes. Without loss of generality we assume again that the set $X$ is full-dimensional. The sequence of polytopes $\left\{P^{r}\right\}_{r \in \mathbb{N}}$ is such that $X \subset P^{r+1} \subset P^{r}$ for all $r \in \mathbb{N}$ and $X=\lim _{r} P^{r}$. For $r \in \mathbb{N}$, let the set $M^{r}$ be defined by

$$
M^{r}=\left\{a \in \mathbb{R}^{n}\left|a=m /\|m\|_{2}, m \in \mathbb{Z}^{n}, 1 \leq \sum_{i=1}^{n}\right| m_{i} \mid \leq r\right\} \cup\{-c, c\} .
$$

The polytope $P^{r}$ is now defined by

$$
P^{r}=\left\{y \in \mathbb{R}^{n} \mid a^{\top} y \leq b(a), a \in M^{r}\right\}, r \in \mathbb{N},
$$

where for $a \in \mathbb{R}^{n} \backslash\left\{0^{n}\right\}$ the number $b(a)$ is given by

$$
b(a)=\max \left\{a^{\top} x \mid x \in X\right\} .
$$

Notice that $b(-c)=0, b(c)=1$, and $P^{r} \subset H$ for all $r \in \mathbb{N}$. For any $a \in \mathbb{R}^{n} \backslash\left\{0^{n}\right\}$, let the hyperplane $H(a)$ be given by $H(a)=\left\{y \in \mathbb{R}^{n} \mid a^{\top} y=b(a)\right\}$ and the halfspace $H^{-}(a)$ by $H^{-}(a)=\left\{y \in \mathbb{R}^{n} \mid a^{\top} y \leq b(a)\right\}$. Then, for every $r \in \mathbb{N}$,

$$
P^{r}=\left\{y \in \mathbb{R}^{n} \mid y \in H^{-}(a) \text { for all } a \in M^{r}\right\}
$$

and $X \subset H^{-}(a)$ for any $a \in M^{r}$, and so $X \subset P^{r}$. Moreover, since $M^{r} \subset M^{r+1}$, we also have $P^{r+1} \subset P^{r}$ for all $r \in \mathbb{N}$.

Lemma 5.1 The $\lim _{r} P^{r}$ is well defined and is equal to the set $X$.

Proof: Since for every $r \in \mathbb{N}$ it holds that $X \subset P^{r+1} \subset P^{r}, \lim _{r} P^{r}$ exists, contains $X$ and is equal to the set $\bigcap_{r \in \mathbb{N}} \operatorname{cl} P^{r}$ where $\operatorname{cl} P^{r}$ denotes the closure of $P^{r}$. See for example Exercise 4.3 of Rockafellar and Wets (1998). By the compactness of every $P^{r}$ the latter set is equal to $\bigcap_{r \in \mathbb{N}} P^{r}$. To show that $X=\bigcap_{r \in \mathbb{N}} P^{r}$, suppose that there exists $y \notin X$ satisfying $y \in P^{r}$ for all $r \in \mathbb{N}$. Since $y \notin X$ and $X$ is a compact, convex set in $\mathbb{R}^{n}$, there exists a hyperplane in $\mathbb{R}^{n}$ strictly separating $y$ from $X$. Let $H^{*}=\left\{z \in \mathbb{R}^{n} \mid h^{\top} z=d\right\}$ for some $h \neq 0$ and $d \in \mathbb{R}$ be such a hyperplane. Without loss of generality all components of $h$ are integer, $h^{\top} y>d$, and $h \neq \beta c$ for any $\beta \in \mathbb{R}$. Let the vector $a$ be defined by $a=h /\|h\|_{2}$. Then $a$ is an element of $M^{r}$ for any $r \geq \sum_{i=1}^{n}\left|h_{i}\right|$. Let $e=d /\|h\|_{2}$, then $H^{*}=\left\{z \in \mathbb{R}^{n} \mid a^{\top} z=e\right\}$ and so $a^{\top} y>e$ and $a^{\top} x<e$ for any $x \in X$. Consequently, 
$b(a)=\max \left\{a^{\top} x \mid x \in X\right\}<e$ and so $a^{\top} y>e>b(a)$. Hence, $y \notin H^{-}(a)$ while $a \in M^{r}$ for any $r \geq \sum_{i=1}^{n}\left|h_{i}\right|$, i.e., $y \notin P^{r}$ for $r$ large enough. This contradicts the fact that $y \in P^{r}$ for all $r \in \mathbb{N}$.

For any $r \in \mathbb{N}$, let the faces $P_{0}^{r}$ and $P_{1}^{r}$ of $P^{r}$ be defined by

$$
P_{0}^{r}=\left\{y \in P^{r} \mid c^{\top} y=0\right\}
$$

and

$$
P_{1}^{r}=\left\{y \in P^{r} \mid c^{\top} y=1\right\} .
$$

Since both $c$ and $-c$ belong to $M^{r}$, it holds that $X^{-} \subset P_{0}^{r}$ and $X^{+} \subset P_{1}^{r}$ for all $r \in \mathbb{N}$. Next take a sequence of simplicial subdivisions $\left\{T^{r}\right\}_{r \in \mathbb{N}}$ such that for every $r \in \mathbb{N}$ the subdivision $T^{r}$ is a triangulation of $P^{r}$ and mesh $T^{r}<r^{-1}$, i.e., every element of $T^{r}$ is an $n$-dimensional simplex in $P^{r}$, being the convex hull of $n+1$ affinely independent points in $P^{r}$ and with diameter less than $r^{-1}$, the union of all elements in $T^{r}$ equals $P^{r}$, and the intersection of any two elements in $T^{r}$ is a common face of both. For triangulations satisfying these properties, we refer to Talman and Yamamoto (1989). Since for any given $r \in \mathbb{N}$, the set $M^{r}$ is finite, we can index the elements of $M^{r}$ from 1 up to, say, $m_{r}$ and assume without loss of generality that $P^{r}$ is simple and has no redundant constraints. So, the polytope $P^{r}$ can be rewritten as

$$
P^{r}=\left\{y \in \mathbb{R}^{n} \mid\left(a^{i, r}\right)^{\top} y \leq b_{i}^{r}, i \in I_{m_{r}}\right\},
$$

where $b_{i}^{r}=b\left(a^{i, r}\right), i \in I_{m_{r}}, r \in \mathbb{N}$. For $I \subset I_{m_{r}}$, define the face $F^{r}(I)$ of $P^{r}$ by

$$
F^{r}(I)=\left\{y \in P^{r} \mid\left(a^{i, r}\right)^{\top} y=b_{i}^{r}, i \in I\right\}
$$

if this set is nonempty. Due to the simpleness of $P^{r}$ and because there are no redundant constraints, we have that if nonempty the dimension of $F^{r}(I)$ equals $n-|I|$, where $|B|$ is the number of elements of a finite set $B$. For $r \in \mathbb{N}$, let $\mathcal{I}^{r}=\left\{I \subset I_{m_{r}} \mid F^{r}(I) \neq \emptyset\right\}$ be the collection of index sets corresponding to the faces of $P^{r}$. For $I \subset \mathcal{I}^{r}, r \in \mathbb{N}$, we also define the dual set $A^{r}(I)$ by

$$
A^{r}(I)=\left\{y \in \mathbb{R}^{n} \mid y=\sum_{h \in I} \mu_{h} a^{h, r}+\beta c, \mu_{h} \geq 0, h \in I, \beta \in \mathbb{R}\right\} .
$$

Since for any $r \in \mathbb{N}, T^{r}$ is a simplicial subdivision of $P^{r}$, every face of $P^{r}$ is simplicially subdivided also. We denote the triangulation of a face $F^{r}(I)$ of $P^{r}$ and induced by $T^{r}$ by $T^{r}(I)$. For any $r \in \mathbb{N}$, the mapping $\phi$ on $X$ is now extended to the mapping $\psi^{r}$ on $P^{r}$ defined by

$$
\psi^{r}(y)=\phi(p(y)), y \in P^{r}
$$


Finally, let $f^{r}$ be the piecewise linear approximation of $\psi^{r}$ on $P^{r}$ with respect to the triangulation $T^{r}$, i.e., if $y=\sum_{j=1}^{n+1} \lambda_{j} y^{j} \in \sigma$ for some simplex $\sigma \in T^{r}$ with vertices $y^{j}$, $j \in I_{n+1}$, where all $\lambda^{j}$ s are nonnegative and sum up to one, then $f^{r}(y)=\sum_{j=1}^{n+1} \lambda_{j} f^{j}$, for some given $f^{j} \in \psi^{r}\left(y^{j}\right), j \in I_{n+1}$. Since $p$ is a continuous function, the mapping $\psi^{r}$ is upper semicontinuous, bounded, nonempty-, convex- and compact-valued on $P^{r}$ and the function $f^{r}$ is continuous on $P^{r}$ and linear on every simplex of $T^{r}$, for every $r \in \mathbb{N}$.

According to the results in Herings, Talman, and Yang (2001), for every $r \in \mathbb{N}$ there exists a piecewise linear path $\pi^{r}([0,1])$ in $P^{r}$ satisfying $\pi^{r}(0) \in P_{0}^{r}, \pi^{r}(1) \in P_{1}^{r}$, and for any $y \in \pi^{r}([0,1])$ there exists $I^{r} \in \mathcal{I}^{r}$ satisfying both $y \in F^{r}\left(I^{r}\right)$ and $f^{r}(y) \in A^{r}\left(I^{r}\right)$, i.e., $y$ is a stationary point of the piecewise linear function $f^{r}$ on $P^{r}$ with respect to the vector c. More precisely, a linear piece of the path $\left.\pi^{r}([0,1])\right)$ lies in a simplex $\tau^{r}$ of $T^{r}\left(I^{r}\right)$ for some $I^{r} \in \mathcal{I}$ and can be generated by making a semi-lexicographic linear programming pivot step with one of the variables in the system of $n+1$ equations and $n+2$ variables

$$
\sum_{j=1}^{t^{r}+1} \lambda_{j}\left(\begin{array}{c}
1 \\
-f^{r}\left(y^{j, r}\right)
\end{array}\right)+\sum_{h \in I^{r}} \mu_{h}\left(\begin{array}{c}
0 \\
a^{h, r}
\end{array}\right)+\beta\left(\begin{array}{l}
0 \\
c
\end{array}\right)=\left(\begin{array}{c}
1 \\
0^{n}
\end{array}\right)
$$

satisfying $\lambda_{j} \geq 0$ for $j \in I_{t^{r}+1}, \mu_{h} \geq 0$ for $h \in I^{r}$, and $\beta \in \mathbb{R}$, where $y^{1, r}, \cdots, y^{t^{r}+1, r}$ are the vertices of the $t^{r}$-dimensional simplex $\tau^{r}$ with $t^{r}=n-\left|I^{r}\right|$. Since for any $r \in \mathbb{N}$ the triangulation $T^{r}$ consists of a finite number of simplices and no simplex in some $T^{r}\left(I^{r}\right)$ can be visited more than once, the path $\pi^{r}([0,1])$ can be generated in this way within a finite number of iterations. Also the point $\pi^{r}(0)$ in $P_{0}^{r}$ can be found within a finite number of iterations, see Herings, Talman, and Yang (2001).

In order to show that the piecewise linear paths $\pi^{r}([0,1]), r \in \mathbb{N}$, can be considered to be an approximation of a connected set of stationary points of $\phi$ on $X$ with respect to $c$, we show that any convergent sequence $\left\{x^{r}\right\}_{r \in \mathbb{N}}$ of points, satisfying $x^{r} \in \pi^{r}([0,1])$ for all $r \in \mathbb{N}$, with limit $x^{*}$ satisfies that the point $x^{*}$ is a stationary point of $\phi$ on $X$ with respect to $c$. From Lemma 5.1 it follows that the limit $x^{*}$ belongs to $X$. Moreover, since $x^{r} \in \pi^{r}([0,1])$, for every $r \in \mathbb{N}$ there exists $I^{r} \in \mathcal{I}^{r}$ satisfying both $x^{r} \in F^{r}\left(I^{r}\right)$ and $f^{r}\left(x^{r}\right) \in A^{r}\left(I^{r}\right)$, i.e.,

(i) $x^{r}=\sum_{j=1}^{t^{r}+1} \lambda_{j}^{r} x^{j, r} \in \tau^{r}$ for some $t^{r}$-dimensional simplex $\tau^{r} \in T^{r}\left(I^{r}\right)$ with vertices $x^{j, r}$, $j \in I_{t^{r}+1}$, where $t^{r}=n-\left|I^{r}\right|$ and the $\lambda_{j}^{r}$ s being nonnegative and summing up to one,

(ii) $f^{r}\left(x^{r}\right)=\sum_{h \in I^{r}} \mu_{h}^{r} a^{h, r}+\beta^{r} c$ for some $\mu_{h}^{r} \geq 0, h \in I^{r}$, and $\beta \in \mathbb{R}$.

Without loss of generality we assume that for every $r \in \mathbb{N}$ it holds that $I^{r}=I_{k}$ for some $k \in\{0\} \cup I_{n}$ (where $I_{0}=\emptyset$ ). Since according to (i) $x^{r} \in F^{r}\left(I_{k}\right.$ ), for all $h \in I_{k}$ we have that $\left(a^{h, r}\right)^{\top} y \leq b_{h}^{r}$ for any $y \in P^{r}, r \in \mathbb{N}$. For every $h \in I_{k}$, the $a^{h, r}$ s lie in a compact set. Therefore, by taking subsequent subsequences if necessary, for every $h \in I_{k}$ the sequence $\left\{a^{h, r}\right\}_{r \in \mathbb{N}}$ converges to some vector $a^{h}$ satisfying $\left\|a^{h}\right\|_{2}=1$. Clearly, by taking limits, 
for every $h \in I_{k}$ it holds that $\left(a^{h}\right)^{\top} x \leq\left(a^{h}\right)^{\top} x^{*}$ for any $x \in X$, so that $a^{h} \in G\left(x^{*}\right)$ for all $h \in I_{k}$. Since $\phi$ is bounded, the solutions of the system of equations on the sequence also lie in a compact set, i.e., by taking subsequences again if necessary, $\lambda_{j}^{r} \rightarrow \lambda_{j} \geq 0$, $j \in I_{t+1}$, with sum equal to 1 , where $t=n-k, \mu_{h}^{r} \rightarrow \mu_{h} \geq 0, h \in I_{k}, \beta^{r} \rightarrow \beta \in \mathbb{R}$, and $f^{r}\left(x^{j, r}\right) \rightarrow f^{j}, j \in I_{t+1}$. Since meshT $T^{r}$ goes to zero, for every $j \in I_{t+1}$ it holds that $x^{j, r} \rightarrow x^{*}$. Now, let $f=\sum_{j=1}^{t+1} \lambda_{j} f^{j}$. From the upper semicontinuity of $\phi$ it follows that $f^{j} \in \phi\left(x^{*}\right)$ for all $j$ and from the convex-valuedness of $\phi$ it follows that $f \in \phi\left(x^{*}\right)$. On the other hand, since $f^{r}\left(x^{r}\right) \rightarrow f$, it follows from (ii) that

$$
f=\sum_{h \in I_{k}} \mu_{h} a^{h}+\beta c
$$

where $\mu_{h} \geq 0, h \in I_{k}$, and $\beta \in \mathbb{R}$. We already showed that $a^{h} \in G\left(x^{*}\right)$ for all $h \in I_{k}$. Hence, using Lemma 2.5 and the fact from Lemma 2.2 that $G\left(x^{*}\right)$ is a convex cone, we obtain that $f \in G\left(x^{*}\right)$. Consequently, $x^{*}$ is a stationary point of $\phi$ on $X$ with respect to $c$.

In case of Theorems 3.2 or 3.6 the piecewise linear approximation $f^{r}$ must be chosen in such a way that $f^{r}(y)$ lies in the supposed nonempty intersection for $p(y)$ in case $y$ is a vertex of a simplex of the triangulation $T^{r}$ of $P^{r}$. For these two theorems it is not necessary to approximate the bigger set $Q$ by a sequence of polytopes. Approximating $X$ by a sequence of polytopes suffices. For Theorem 3.1 any piecewise linear approximation of $\phi$ can be chosen. For all three theorems it holds that the limit point $x^{*}$ is a zero point of $\phi$ in $X$. Notice that in this way we also obtain a constructive proof of the existence of a continuum of zero points of a mapping on a compact, convex set.

\section{References}

[1] E.L. Allgower and K. Georg, Numerical Continuation Methods: An Introduction, Springer-Verlag, Berlin, 1990.

[2] J.P. Aubin and A. Cellina, Differential Inclusions, Springer-Verlag, Berlin, 1984.

[3] L.E.J. Brouwer, "Über Abbildung von Mannigfaltigkeiten", Mathematische Annalen $71(1912)$ 97-115.

[4] F.E. Browder, "On continuity of fixed points under deformation of continuous mapping", Summa Brasiliensis Mathematicae 4 (1960) 183-191.

[5] D.J. Brown, P.M. DeMarzo and B.C. Eaves, "Computing zeroes of sections of vector bundles using homotopies and relocalization", Mathematics of Operations Research 21 (1996) 26-43. 
[6] P.M. DeMarzo and B.C. Eaves, "Computing equilibria of GEI by relocalization on a Grassmann manifold", Journal of Mathematical Economics 26 (1996) 479-497.

[7] B.C. Eaves, "On the basic theory of complementarity", Mathematical Programming 1 (1971) 68-75.

[8] B.C. Eaves, "Homotopies for computation of fixed points", Mathematical Programming 3 (1972) 1-22.

[9] J. Freidenfelds, "A set intersection theorem and applications", Mathematical Programming 7 (1974) 199-211.

[10] W.W. Hogan, "Point-to-set maps in mathematical programming", SIAM Review 15 (1973) 591-603.

[11] P.J.J. Herings, "On the Existence of a Continuum of Constrained Equilibria", Journal of Mathematical Economics 30 (1998) 257-273.

[12] P.J.J. Herings and A.J.J. Talman, "Intersection theorems with a continuum of intersection points", Journal of Optimization Theory and Applications 96 (1998) 311-335.

[13] P.J.J. Herings, A.J.J. Talman and Z. Yang, "The computation of a continuum of constrained equilibria", Mathematics of Operations Research 21 (1996) 675-696.

[14] P.J.J. Herings, A.J.J. Talman and Z. Yang, "Variational inequality problems with a continuum of solutions: existence and computation", SIAM Journal on Control and Optimization 39 (2001) 1852-1873.

[15] S. Kakutani, "A generalization of Brouwer's fixed point theorem", Duke Mathematical Journal 8 (1941) 457-459.

[16] B. Knaster, C. Kuratowski, and C. Mazurkiewicz, "Ein Beweis des Fixpunktsatzes für n-dimensionale Simplexe", Fundamenta Mathematicae 14 (1929) 132-137.

[17] G. van der Laan and A.J.J. Talman, "A restart algorithm for computing fixed points without an extra dimension", Mathematical Programming 17 (1979) 74-84.

[18] G. van der Laan, A.J.J. Talman, and Z. Yang, "Existence and approximation of robust solutions of variational inequality problems over polytopes", SIAM Journal on Control and Optimization 37 (1998) 333-352.

[19] A. Mas-Colell, "A note on a theorem of F. Browder", Mathematical Programming 6 (1974) 229-233. 
[20] R.T. Rockafellar and R.J-B. Wets, Variational Analysis, Springer-Verlag, Berlin, 1998.

[21] H. Scarf, "The approximation of fixed points of a continuous mapping", SIAM Journal on Applied Mathematics 15 (1967) 1328-1343.

[22] A.J.J. Talman and Y. Yamamoto, "A simplicial algorithm for stationary point problems on polytopes", Mathematics of Operations Research 14 (1989) 383-399.

[23] Y. Yamamoto, "A path-following procedure to find a proper equilibrium of finite games", International Journal of Game Theory 22 (1993) 249-259.

[24] Z. Yang, "A simplicial algorithm for computing robust stationary points of a continuous function on the unit simplex", SIAM Journal on Control and Optimization 34 (1996) 191-206.

[25] Z. Yang, Computing Equilibria and Fixed Points, Kluwer Academic Publishers, Boston, 1999. 